\title{
Predicting the strain-mediated topological phase transition in 3D cubic $\mathrm{ThTaN}_{3}$
}

\author{
Chunmei Zhang and Aijun Du*
}

\author{
Full Research Paper \\ Address: \\ School of Chemistry, Physics and Mechanical Engineering, \\ Queensland University of Technology, Gardens Point Campus, QLD \\ 4001, Brisbane, Australia \\ Email: \\ Aijun Du* - aijun.du@qut.edu.au \\ * Corresponding author \\ Keywords: \\ Dirac cone; strain; $\mathrm{ThTaN}_{3}$; topological insulator
}

Beilstein J. Nanotechnol. 2018, 9, 1399-1404. doi:10.3762/bjnano.9.132

\author{
Received: 01 December 2017 \\ Accepted: 25 April 2018 \\ Published: 11 May 2018 \\ This article is part of the Thematic Series "Topological materials". \\ Guest Editor: J. J. Palacios \\ (C) 2018 Zhang and Du; licensee Beilstein-Institut. \\ License and terms: see end of document.
}

\begin{abstract}
The cubic $\mathrm{ThTaN}_{3}$ compound has long been known as a semiconductor with a band gap of approximately $1 \mathrm{eV}$, but its electronic properties remain largely unexplored. By using density functional theory, we find that the band gap of $\mathrm{ThTaN}_{3}$ is very sensitive to the hydrostatic pressure/strain. A Dirac cone can emerge around the $\Gamma$ point with an ultrahigh Fermi velocity at a compressive strain of $8 \%$. Interestingly, the effect of spin-orbital coupling (SOC) is significant, leading to a band gap reduction of $0.26 \mathrm{eV}$ in the $\mathrm{ThTaN}_{3}$ compound. Moreover, the strong SOC can turn $\mathrm{ThTaN}_{3}$ into a topological insulator with a large inverted gap up to $0.25 \mathrm{eV}$, which can be primarily attributed to the inversion between the d-orbital of the heavy element Ta and the p-orbital of $\mathrm{N}$. Our results highlight a new $3 \mathrm{D}$ topological insulator with strain-mediated topological transition for potential applications in future spintronics.
\end{abstract}

\section{Introduction}

The $\mathrm{ThTaN}_{3}$ compound generally presents three structural phases in cubic perovskite (c-PV), hypothetic orthorhombic perovskite (o-PV $\mathrm{GdFeO}_{3}$-type), and post-perovskite (PPV) forms [1]. Among them, c-PV ThTaN 3 was first synthesized in early 1995 [2] and is known to crystallize in the space group $P m 3 m$ with a band gap of approximately $1 \mathrm{eV}$ [1]. Pressure can induce a phase transition from c-PV to o-PV and PPV accompanied by the transition from a moderate band gap semiconductor $(\approx 1 \mathrm{eV}$ band gap in c-PV) to a small band gap semiconductor (PPV) in $\mathrm{ThTaN}_{3}$ [1]. c-PV $\mathrm{ThTaN}_{3}$ has also been proposed as a potential ground for studying nonlinear optical response [2] due to its large band gap and non-centrosymmetry. As protons are found to be significantly stable in nitrides, c-PV $\mathrm{ThTaN}_{3}$ is also evaluated as an ideal proton-conducting ceramic [1]. Nevertheless, theoretical understanding of the electronic properties of $\mathrm{ThTaN}_{3}$ is so far very limited and mainly focused on pressure-induced phase transition [3]. Therefore, a systematic study of the electron structure of $\mathrm{ThTaN}_{3}$ in a certain phase is highly desired.

Topological insulators (TIs) have attracted much attention due to their distinct quantum mechanical properties, which makes 
them important in the fields of physics [4,5], chemistry, and materials science [6]. TIs are materials with a bulk band gap generated by strong spin-orbit coupling (SOC) that have topologically protected metallic surface states. Although many materials are theoretically predicted to be TIs [7-11], the experimental realization of TIs is very limited. Therefore, the search for experimentally synthesized large band gap TIs is of paramount importance for their practical application. Theoretically, the transition from the trival insulator to the topological insulator can be achieved by increasing the SOC or by altering the lattice parameters [12,13]. A number of compounds [14-25], such as LaPtBi, LuPtSb, YPdBi [15-18], and HgTe [19,20], have been studied using a first-principles approach, showing that they can be turned into TIs under external strain. All these materials possess heavy elements and the strong SOC can induce a band inversion, which is a typical mechanism for TIs $[26,27]$.

The experimentally observed pressure-induced phase transition in $\mathrm{ThTaN}_{3}$ indicates that the electronic structure of $3 \mathrm{D} \mathrm{ThTaN}$ is likely very sensitive to the external strain. In particular, c-PV $\mathrm{ThTaN}_{3}$ can crystallize in the tetragonal shape with $\mathrm{C}_{4}$ rotational symmetry, which is an ideal platform to study its topological properties [28]. The combination of such $\mathrm{C}_{4}$ rotational and time-reversal symmetry and the heavy elements (Th, Ta) in $\mathrm{ThTaN}_{3}$ are expected to substantially alter the electronic band structure and thus achieve an exotic topological property [26].

By using first-principles calculations, we demonstrate here, for the first time, that the cubic perovskite $\mathrm{ThTaN}_{3}$, a relatively large band gap semiconductor, can turn into a TI under moderate pressure/strain. A Dirac cone can emerge in the $\mathrm{ThTaN}_{3}$ compound with an ultrahigh Fermi velocity under an $8 \%$ compressive strain. The band gap opening, induced by SOC, can be as high as $0.25 \mathrm{eV}$, which is large enough for the realization of the quantum spin Hall (QSH) states at room temperature. In addition, by tuning the SOC strength, we predict that the topological feature actually starts to show up at a 5\% compressive strain. The strain-mediated topological phase transition in the perovskite $\mathrm{ThTaN}_{3}$ compound is attributed to band inversion between the d-orbital of the heavy elements and the p-orbital of the $\mathrm{N}$ atom $[12,29,30]$.

\section{Computational Methods}

First-principles calculations were performed based on density functional theory (DFT) as implemented in the plane wave basis VASP code [31-33]. A generalized gradient approximation (GGA) in the Perdew, Burke, and Ernzerhof (PBE) form exchange-correlation functional was used. The hybrid Heyd-Scuseria-Ernzerhof (HSE06) functional [34,35] was adopted for the accurate calculation of band structures of 3D $\mathrm{ThTaN}_{3}$. A plane-wave basis set with an energy cut-off of $500 \mathrm{eV}$ was employed and long range van der Waals dispersion [36] was incorporated to correct the total energy. The geometry structures were fully optimized until the maximum energy and force were less than $10^{-6} \mathrm{eV}$ and $0.01 \mathrm{eV} / \AA$, respectively. A Monkhorst-Pack $k$-point mesh of $7 \times 7 \times 7$ was used for geometry optimization. The SOC effect was also considered in the calculation. The electron effective mass $\left(m^{*}\right)$ of $\mathrm{ThTaN}_{3}$ at the conduction band minimum (CBM) is estimated from the curvature of the electronic band dispersion, that is, the formula

$$
m^{*}=\hbar\left(\frac{\partial E^{2}}{\partial k^{2}}\right)^{-1}
$$

where $E$ and $k$ are the band energy and reciprocal lattice vector. For anisotropic materials, $m^{*}=\sqrt{m_{i}^{*} m_{j}^{*} m_{k}^{*}}$, where $i, j$ and $k$ label the transport direction along the $x, y$ and $z$-axis.

\section{Results and Discussion}

The geometry structure of cubic perovskite $\mathrm{ThTaN}_{3}$ was first fully relaxed as shown in Figure 1a. It crystallizes in the space group $P m 3 m$ with $\mathrm{C}_{4}$ rotational symmetry. The lattice parame- (a) crystal structure

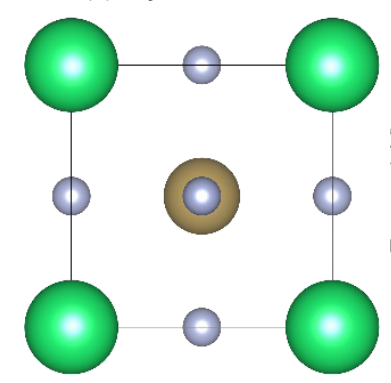

(b) PBE

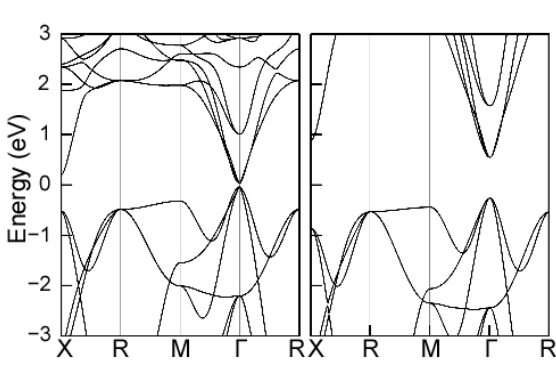

(d) $\mathrm{PBE}+\mathrm{SOC}$

(e) HSE+SOC

Figure 1: (a) Top view of $\mathrm{ThTaN}_{3}$ with green, grey, and brown spheres representing $\mathrm{Th}, \mathrm{N}$, and Ta atoms, respectively. (b-e) The band structures of 3D cubic $\mathrm{ThTaN}_{3}$ calculated by PBE, HSE, PBE+SOC, and HSE+SOC methods, respectively. 
ters of c-PV ThTaN 3 were then calculated by using the PBE functional and the hybrid HSE06 functional methods, respectively. It was found that the PBE functional overestimates the experimental lattice constants by $1 \%$, whereas the HSE06 can successfully reproduce the experimentally reported lattice parameters (4.02 $\AA)$ [2].

Figure 1 presents the detailed electronic band structure of 3D $\mathrm{ThTaN}_{3}$ for the PBE exchange correlation (Figure 1b) and HSE hybrid functional (Figure 1c). A small direct band gap of $0.07 \mathrm{eV}$ at the $\Gamma$ point is predicted by the PBE functional and the HSE functional produced a large band gap of $0.76 \mathrm{eV}$, which is very close to the experimental measurement $(1.0 \mathrm{eV})$ [1]. The band gap should exhibit substantial differences depending on the relative weights of the Hartree-Fock and traditional LDA or GGA exchange energies in the hybrid functional as well as those of the long range van der Waals interactions. However, we found that the impact of van der Waals interaction on the band gap of $\mathrm{ThTaN}_{3}$ is negligible. Figure $1 \mathrm{~d}$ and Figure 1e present band structures in the presence of the effect of SOC. Clearly, an energy gap of $0.15 \mathrm{eV}$ and $0.49 \mathrm{eV}$ were opened by the SOC for the PBE and the HSE functional methods, respectively. Compared to the HSE result without SOC (Figure 1c), the band gap reduction is significantly high $(0.26 \mathrm{eV})$ after the incorporation of SOC.

Then we turned to study the effect of strain [37] on the electronic structure of c-PV $\mathrm{ThTaN}_{3}$ by applying a hydrostatic strain ranging from $-10 \%$ (compressive strain) to $+15 \%$ (tensile strain) on $3 \mathrm{D} \mathrm{ThTaN}_{3}$. As shown in Figure $2 \mathrm{~d}-\mathrm{g}$, the size of the direct gap continued to increase as the positive strain was increased. At a strain of $3 \%$, the direct band gap turned to an indirect one and the band gap slightly decreased with further increasing strain. When a compressive strain was exerted into 3D $\mathrm{ThTaN}_{3}$, the band gap could be significantly reduced. As shown in Figure 2a, the energy gap was reduced to $0 \mathrm{eV}$ at a compressive strain of $-8 \%$. A Dirac-cone-like band structure [38] emerged with an ultrahigh Fermi velocity $6.33 \times 10^{5} \mathrm{~m} / \mathrm{s}$ that is comparable to that of graphene $\left(1.1 \times 10^{6} \mathrm{~m} / \mathrm{s}\right)$ [39]. It is very important to note that the conduction band (CB) of $\mathrm{ThTaN}_{3}$ is very dispersive around the $\Gamma$ point, signifying a very low electron effective mass. The effective mass of the electron at the $\Gamma$ point is calculated to be $0.395 m_{\mathrm{e}}$. Such a small electron mass will greatly improve charge carrier mobility, suggesting great potential for application of $\mathrm{ThTaN}_{3}$ in electronics.

As eluded to above, the effect of SOC on the band gap of $\mathrm{ThTaN}_{3}$ is significant. It is therefore important to further study the effect of strain on the electronic structure of $\mathrm{ThTaN}_{3}$ in the presence of SOC (Figure 3). For strain-free $\mathrm{ThTaN}_{3}$, the band gap is $0.49 \mathrm{eV}$ as calculated by the HSE+SOC method. The band gap is reduced approximately $0.26 \mathrm{eV}$ compared to the HSE result $(0.76 \mathrm{eV})$ without SOC. When an $8 \%$ compressive strain is exerted on the $\mathrm{ThTaN}_{3}$ compound, SOC opens a large band gap (approximately $0.25 \mathrm{eV}$ ) for the Dirac cone as shown in Figure 3d. It can be seen that under compressive strain, the SOC gap of $\mathrm{ThTaN}_{3}$ can be closed and reopened. In addition, the shape of the band structure is changed correspondingly, indicating a topological phase transition [40-42]. In order to determine topological features, we calculated the $\mathrm{Z} 2$ topological index $[19,27]$. The topological invariant $Z_{2}$ is $1 ;(0,0,0)$ for $\mathrm{ThTaN}_{3}$ under $8 \%$ compressive strain, which indicates the strong topological property (more details including methods and parities of the relevant bands can be found in Supporting Information File 1). We further scrutinized the SOC band structure of $\mathrm{ThTaN}_{3}$ (Figure 3c) and find that the band inversion actually occurred at a $5 \%$ compressive strain. The above results clearly indicated that we can turn $\mathrm{ThTaN}_{3}$ into a TI by applying an external hydrostatic pressure in the presence of SOC.

We further analyzed the orbital-resolved band structure of $\mathrm{ThTaN}_{3}$ at a lower compressive strain (5\%) as shown in Figure 4. The conduction band (CB) state mainly consisted of $\mathrm{p}_{\mathrm{x}}, \mathrm{p}_{\mathrm{y}}$ and $\mathrm{p}_{\mathrm{z}}$ orbitals of the $\mathrm{N}$ atom, while the valence band

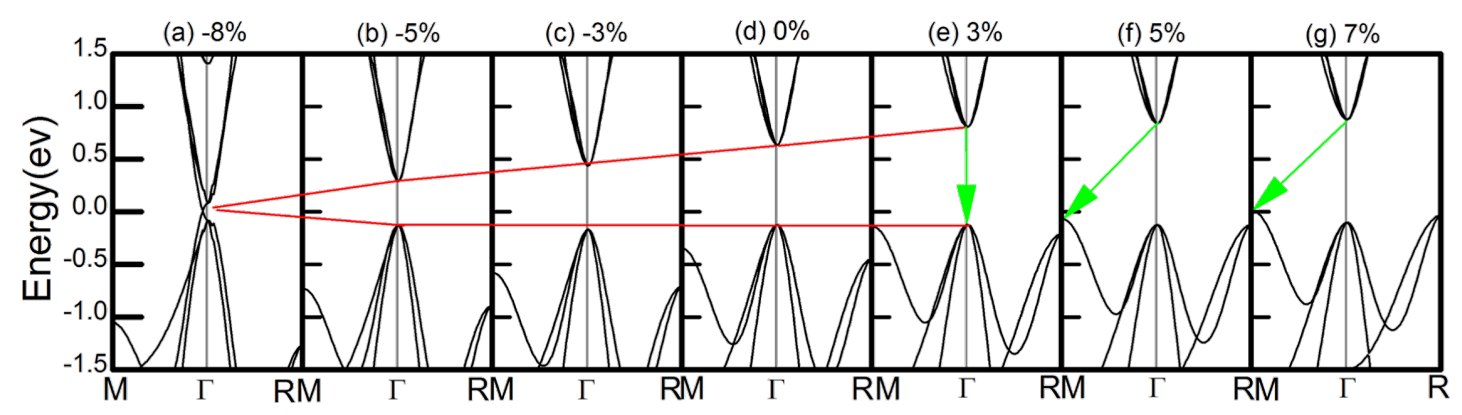

Figure 2: The modulation of band gap (red line) by hydrostatic and tensile strain $(-8 \%$ to $+7 \%)$ in $\mathrm{ThTaN}_{3}$ by using the HSE method. A Dirac cone emerges when a $-8 \%$ strain is added to the $\mathrm{ThTaN}_{3}$ compound. And the green arrow shows that the change from a direct band gap to an indirect band gap with strain is increased from $3 \%$ to $7 \%$. The Fermi level is set at an energy of zero. 


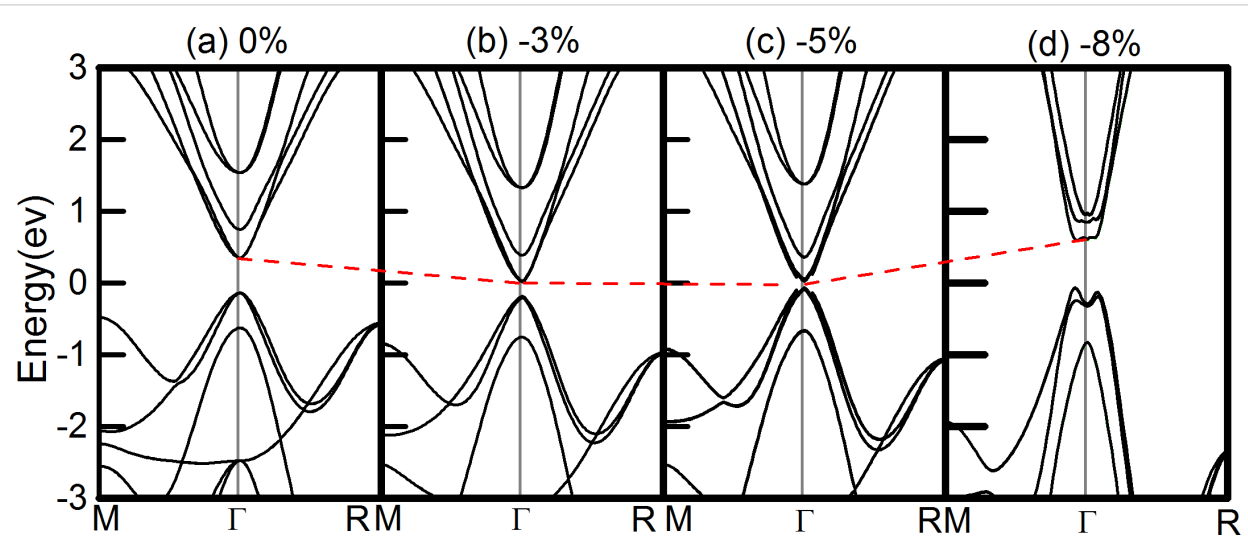

Figure 3: Band structures of $\mathrm{ThTaN}_{3}$ calculated by the $\mathrm{HSE}+\mathrm{SOC}$ method under a strain of $-8 \%$ to $0 \%$. The Fermi level is set to zero.

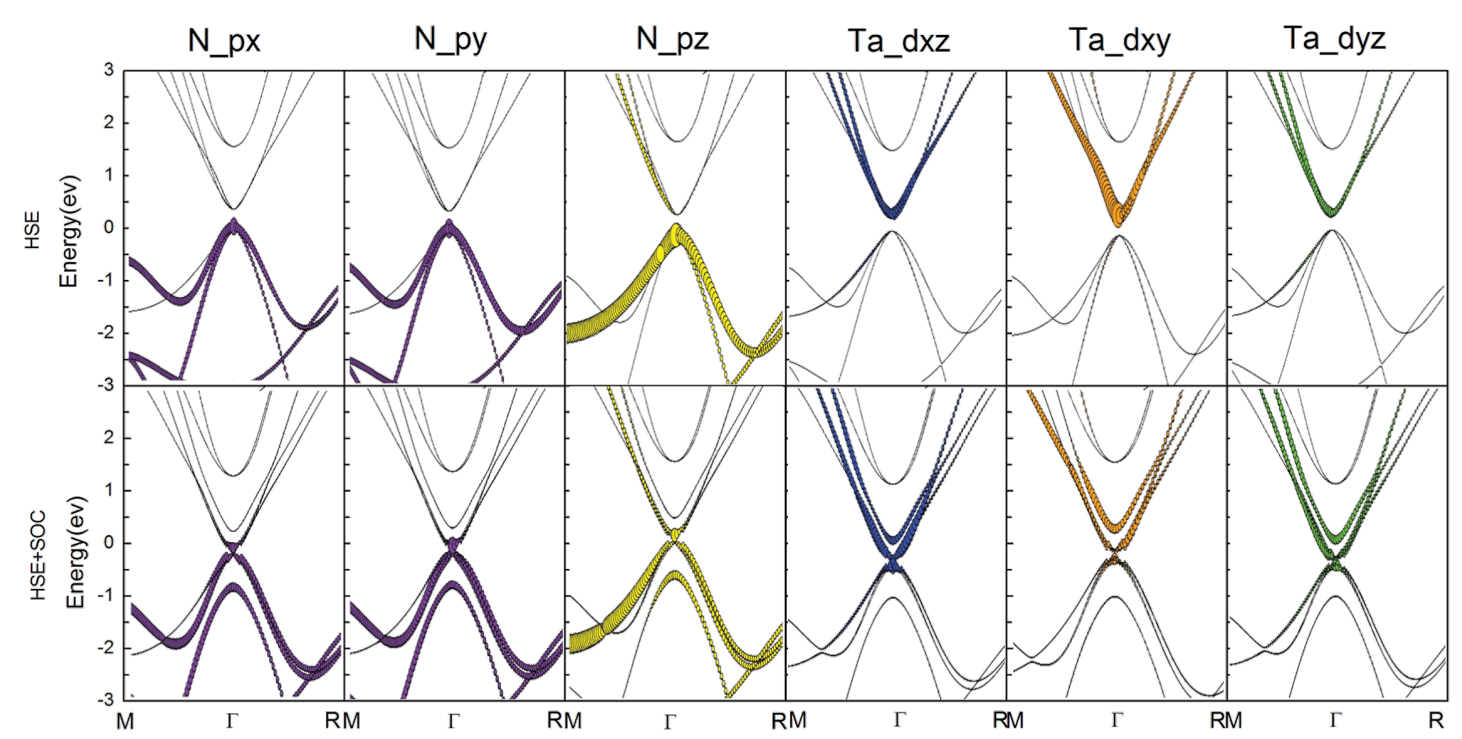

Figure 4: Orbital-resolved band structures for $\mathrm{ThTaN}_{3}$ under $5 \%$ compressive strain as calculated by the HSE (top panel) and HSE+SOC methods (bottom panel). The contributions are mainly from $p_{x}, p_{y}, p_{z}$ of $N$ atoms and $d_{x z}, d_{x y}, d_{y z}$ of Ta atoms. The thicker and thinner lines account for large and low orbital contributions, respectively.

(VB) state is comprised of the hybridization $d_{x y}, d_{x z}, d_{y z}$ orbitals of the Ta atom. When the SOC effect was incorporated, a p-d-type band inversion took place with the reduction of the band gap, manifesting a topological phase transition due to the synergistic effects of SOC and lattice strain.

Generally, the topological phase transition can be also directly observed by modifying the SOC strength $[29,30]$. To provide a clear picture of the band inversion or topological phase transition in $\mathrm{ThTaN}_{3}$ under a 5\% compressive strain, we then studied the electronic structure of $3 \mathrm{D}$ cubic perovskite $\mathrm{ThTaN}_{3}$ at various SOC strengths as shown in Figure 5a-e. With increasing SOC, the VBM and CBM gradually become closer (see Figure $5 \mathrm{a}-\mathrm{e}$ ), and then the gap closed and reopened with a large portion of band inversion, which can be clearly seen from the enormous change of the CBM and VBM band shape into Mexican-hat-like band dispersion, a typical indication of topological phase transition (see Figure 5e).

\section{Conclusion}

In summary, we have systematically studied the electronic structure in the $3 \mathrm{D}$ perovskite $\mathrm{ThTaN}_{3}$ compound. We find the band gap of $\mathrm{ThTaN}_{3}$ is very sensitive to the lattice strain. A Dirac-cone-like band with an ultrahigh Fermi velocity can emerge at a compressive strain of $8 \%$. The topological phase transition can be realized with a large gap $(\approx 0.25 \mathrm{eV})$ opened in the presence of SOC. Further analysis of orbital contribution indicates $\mathrm{p}-\mathrm{d}$ band inversion in $3 \mathrm{D} \mathrm{ThTaN}_{3}$. Our results highlight a new, interesting, 3D, topological insulator material with great potential for future application in spintronics. 


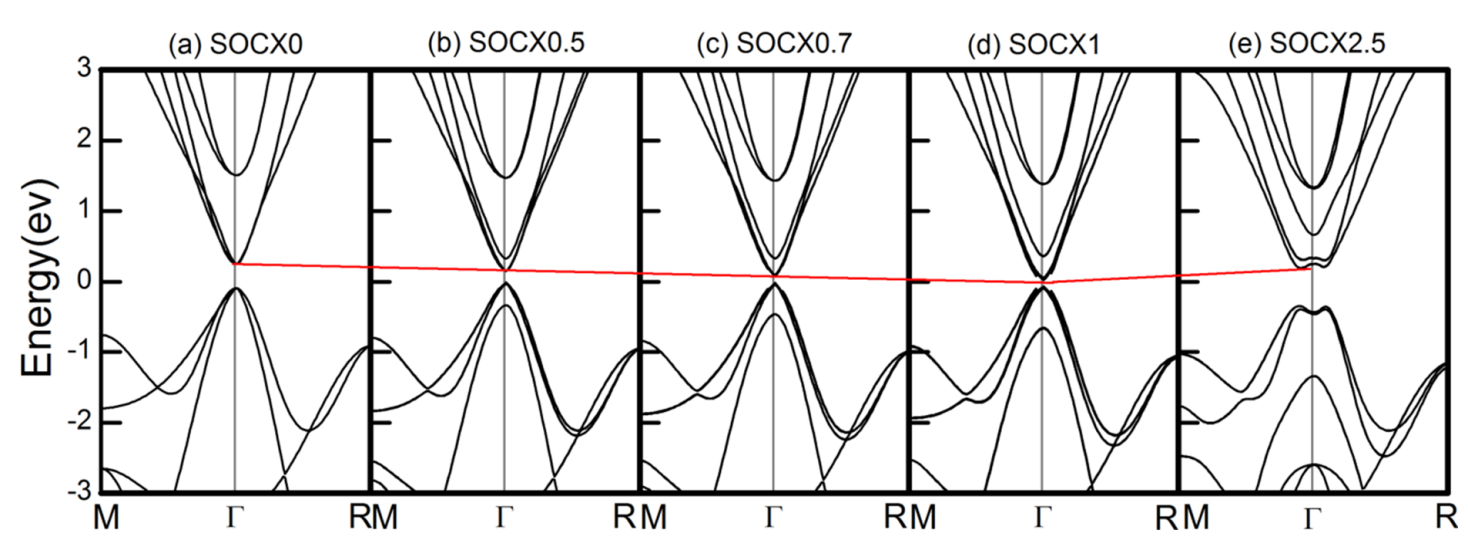

Figure 5: Band structures for 3D cubic $\mathrm{ThTaN}_{3}$ with a $5 \%$ compressive strain as calculated by the HSE+SOC method at different SOC strengths (0-2.5 times). The Fermi energy level is set to zero.

\section{Supporting Information}

\section{Supporting Information File 1}

Additional calculations.

The lattice parameters of $\mathrm{ThTaN}_{3}$ under strain, the surface state of $\mathrm{ThTaN}_{3}$, and the calculation of the topological invariant number Z2.

[https://www.beilstein-journals.org/bjnano/content/ supplementary/2190-4286-9-132-S1.pdf]

\section{Acknowledgements}

We acknowledge generous grants of high-performance computer time from the computing facility at the Queensland University of Technology, The Pawsey Supercomputing Centre and Australian National Facility. A.D. greatly appreciates the Australian Research Council QEII Fellowship (DP110101239) and financial support of Australian Research Council under Discovery Project (DP130102420 and DP170103598).

\section{References}

1. Matar, S. F.; Demazeau, G. J. Solid State Chem. 2010, 183, 994-999. doi:10.1016/j.jssc.2010.03.002

2. Brese, N. E.; DiSalvo, F. J. J. Solid State Chem. 1995, 120, 378-380. doi:10.1006/jssc.1995.1423

3. Polfus, J. M.; Haugsrud, R. Solid State Commun. 2012, 152, 1921-1923. doi:10.1016/j.ssc.2012.07.010

4. Bernevig, B. A.; Zhang, S.-C. Phys. Rev. Lett. 2006, 96, 106802. doi:10.1103/PhysRevLett.96.106802

5. Huang, C.; Zhou, J.; Wu, H.; Deng, K.; Jena, P.; Kan, E. Phys. Rev. B 2017, 95, 045113. doi:10.1103/PhysRevB.95.045113

6. Kong, D.; Cui, Y. Nat. Chem. 2011, 3, 845-849. doi:10.1038/nchem.1171

7. Zhou, L.; Shao, B.; Shi, W.; Sun, Y.; Felser, C.; Yan, B.; Frauenheim, T. 2D Mater. 2016, 3, 035022. doi:10.1088/2053-1583/3/3/035022
8. Zhou, L.; Shi, W.; Sun, Y.; Shao, B.; Felser, C.; Yan, B.; Frauenheim, T. 2D Mater. 2016, 3, 035018. doi:10.1088/2053-1583/3/3/035018

9. Zhou, L.; Kou, L.; Sun, Y.; Felser, C.; Hu, F.; Shan, G.; Smith, S. C.; Yan, B.; Frauenheim, T. Nano Lett. 2015, 15, 7867-7872. doi:10.1021/acs.nanolett.5b02617

10. Ma, Y.; Kou, L.; Dai, Y.; Heine, T. Phys. Rev. B 2016, 94, 201104. doi:10.1103/PhysRevB.94.201104

11. Ma, Y.; Kou, L.; Li, X.; Dai, Y.; Heine, T. NPG Asia Mater. 2016, 8, e264. doi:10.1038/am.2016.51

12. Xu, S.-Y.; Xia, Y.; Wray, L. A.; Jia, S.; Meier, F.; Dil, J. H.; Osterwalder, J.; Slomski, B.; Bansil, A.; Lin, H.; Cava, R. J.; Hasan, M. Z. Science 2011, 332, 560-564. doi:10.1126/science. 1201607

13. Huang, C.; Zhou, J.; Wu, H.; Deng, K.; Jena, P.; Kan, E. J. Phys. Chem. Lett. 2016, 7, 1919-1924. doi:10.1021/acs.jpclett.6b00651

14. Zhou, D.; Li, Q.; Ma, Y.; Cui, Q.; Chen, C. J. Phys. Chem. C 2013, 117, 8437-8442. doi:10.1021/jp401928j

15. Xiao, D.; Yao, Y.; Feng, W.; Wen, J.; Zhu, W.; Chen, X.-Q.; Stocks, G. M.; Zhang, Z. Phys. Rev. Lett. 2010, 105, 096404. doi:10.1103/PhysRevLett.105.096404

16. Feng, W.; Xiao, D.; Zhang, Y.; Yao, Y. Phys. Rev. B 2010, 82, 235121. doi:10.1103/PhysRevB.82.235121

17. Chadov, S.; Qi, X.; Kübler, J.; Fecher, G. H.; Felser, C.; Zhang, S. C. Nat. Mater. 2010, 9, 541-545. doi:10.1038/nmat2770

18. Lin, H.; Wray, L. A.; Xia, Y.; Xu, S.; Jia, S.; Cava, R. J.; Bansil, A.; Hasan, M. Z. Nat. Mater. 2010, 9, 546-549. doi:10.1038/nmat2771

19. Fu, L.; Kane, C. L. Phys. Rev. B 2007, 76, 045302. doi:10.1103/PhysRevB.76.045302

20. Brüne, C.; Liu, C. X.; Novik, E. G.; Hankiewicz, E. M.; Buhmann, H.; Chen, Y. L.; Qi, X. L.; Shen, Z. X.; Zhang, S. C.; Molenkamp, L. W. Phys. Rev. Lett. 2011, 106, 126803. doi:10.1103/PhysRevLett.106.126803

21. Zhang, H.; Ma, Y.; Chen, Z. Nanoscale 2015, 7, 19152-19159. doi:10.1039/C5NR05006E

22. Zhang, S.; Xie, M.; Cai, B.; Zhang, H.; Ma, Y.; Chen, Z.; Zhu, Z.; Hu, Z.; Zeng, H. Phys. Rev. B 2016, 93, 245303. doi:10.1103/PhysRevB.93.245303

23. Ma, Y.; Kou, L.; Li, X.; Dai, Y.; Smith, S. C.; Heine, T. Phys. Rev. B 2015, 92, 085427. doi:10.1103/PhysRevB.92.085427 
24. Zhao, M.; Chen, X.; Li, L.; Zhang, X. Sci. Rep. 2015, 5, 8441. doi:10.1038/srep08441

25. Zhao, M.; Zhang, X.; Li, L. Sci. Rep. 2015, 5, 16108. doi:10.1038/srep16108

26. Bernevig, B. A.; Hughes, T. L.; Zhang, S.-C. Science 2006, 314, 1757-1761. doi:10.1126/science.1133734

27. Fu, L.; Kane, C. L.; Mele, E. J. Phys. Rev. Lett. 2007, 98, 106803. doi:10.1103/PhysRevLett.98.106803

28. Fu, L. Phys. Rev. Lett. 2011, 106, 106802. doi:10.1103/PhysRevLett.106.106802

29. Zhang, W.; Yu, R.; Zhang, H.-J.; Dai, X.; Fang, Z. New J. Phys. 2010 , 12, 065013. doi:10.1088/1367-2630/12/6/065013

30. Murakami, S. Prog. Theor. Phys. Suppl. 2008, 176, 279-301. doi:10.1143/PTPS.176.279

31. Kresse, G.; Furthmüller, J. Comput. Mater. Sci. 1996, 6, 15-50. doi:10.1016/0927-0256(96)00008-0

32. Kresse, G.; Furthmüller, J. Phys. Rev. B 1996, 54, 11169. doi:10.1103/PhysRevB.54.11169

33. Blöchl, P. E. Phys. Rev. B 1994, 50, 17953. doi:10.1103/PhysRevB.50.17953

34. Heyd, J.; Scuseria, G. E.; Ernzerhof, M. J. Chem. Phys. 2003, 118, 8207-8215. doi:10.1063/1.1564060

35. Perdew, J. P.; Burke, K.; Ernzerhof, M. Phys. Rev. Lett. 1996, 77, 3865. doi:10.1103/PhysRevLett.77.3865

36. Grimme, S. J. Comput. Chem. 2006, 27, 1787-1799. doi:10.1002/jcc. 20495

37.Zhang, C.; Kou, L.; He, T.; Jiao, Y.; Liao, T.; Bottle, S.; Du, A. Comput. Mater. Sci. 2018, 149, 158-161. doi:10.1016/j.commatsci.2018.03.027

38. Zhang, C.; Jiao, Y.; Ma, F.; Bottle, S.; Zhao, M.; Chen, Z.; Du, A. Phys. Chem. Chem. Phys. 2017, 19, 5449-5453. doi:10.1039/C7CP00157F

39. Trevisanutto, P. E.; Giorgetti, C.; Reining, L.; Ladisa, M.; Olevano, V. Phys. Rev. Lett. 2008, 101, 226405. doi:10.1103/PhysRevLett.101.226405

40. Zhang, H.; Liu, C.-X.; Qi, X.-L.; Dai, X.; Fang, Z.; Zhang, S.-C. Nat. Phys. 2009, 5, 438-442. doi:10.1038/nphys 1270

41. Xu, Y.; Yan, B.; Zhang, H.-J.; Wang, J.; Xu, G.; Tang, P.; Duan, W.; Zhang, S.-C. Phys. Rev. Lett. 2013, 111, 136804. doi:10.1103/PhysRevLett.111.136804

42. Si, C.; Liu, J.; Xu, Y.; Wu, J.; Gu, B.-L.; Duan, W. Phys. Rev. B 2014, 89, 115429. doi:10.1103/PhysRevB.89.115429

\section{License and Terms}

This is an Open Access article under the terms of the Creative Commons Attribution License

(http://creativecommons.org/licenses/by/4.0), which permits unrestricted use, distribution, and reproduction in any medium, provided the original work is properly cited.

The license is subject to the Beilstein Journal of Nanotechnology terms and conditions:

(https://www.beilstein-journals.org/bjnano)

The definitive version of this article is the electronic one which can be found at:

doi:10.3762/bjnano.9.132 